\title{
Torque ripples in stepping motor driven systems
}

\author{
Stijn Derammelaere, Bram Vervisch, Florian Verbelen, Kurt Stockman \\ Department of Industrial System and Product Design, Ghent University \\ campus Kortrijk 8530, Belgium \\ Email: stijn.derammelaere@ugent.be
}

\section{Keywords}

$<<$ Permanent magnet motor $>>,<<$ Electrical machine $>>,<<$ Motion control $>>,<<$ Noise $>>$

\begin{abstract}
Stepping motor operation is characterized by torque ripples. In this paper it is shown these torque ripples are caused by both the stepping motor drive algorithms and the toothed construction of rotor and stator of the studied hybrid stepping motors. These torque ripples are analyzed, discussed and measured. The torque ripples are measured in the complete operating range of the motor and depicted in this paper for full- half- and micro-stepping. By doing this, the paper provides insight in the vibrating behavior of a stepping motor driven system and possible solutions to overcome this are placed in the right perspective.
\end{abstract}

\section{Introduction}

The two-phase hybrid stepping motor principle is illustrated in Fig. 1 [1]. The stator is equipped with concentrated windings while the multi toothed rotor is magnetized by means of permanent-magnets. The rotor teeth are attracted by the excited stator phase. When a new full-step command pulse is given, the excitation of one phase is released while a second phase is excited. For half- and micro-stepping algorithms, two phases are excited simultaneously in order to increase the number of rotor-position steps in a single revolution.

By counting the step command pulses, open-loop positioning is achieved. The absence of an expensive position sensor, makes these motors very appealing for industrial and domestic applications. Unfortunately, these widely used open-loop drive algorithms result in large torque ripples and possible resonance problems limiting the range of operation [2]. Some drive algorithms described in literature minimize these vibrations but do require some kind of position feedback [3]. As a result, the large majority of the stepping motors in industry are still driven in open-loop using a full-, half- or micro-stepping algorithm. In this paper, the origin of the torque ripples and according torsional vibrations are discussed. In section

an expression describing the generated motor torque is theoretically derived. This expression is used both to gain insight and to model the stepping motor behavior. Simulations based on this model are used to study the impact of both the drive algorithm in section and the stepping motor construction in section . Finally measurement results quantifying these vibrations in the complete operating range of the motor for full-, half- and micro-stepping are discussed in section. Altogether these results provide insight on the vibrating stepping motor behaviour and places these phenomena in the right perspective.



(a) Full-Step principle

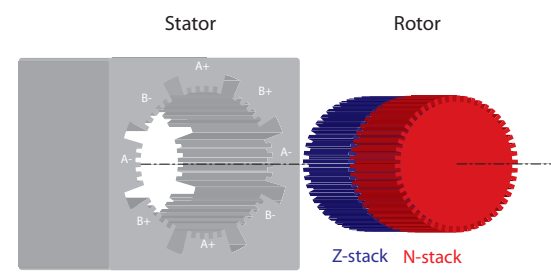

(b) Hybrid stepper motor construction

Figure 1: Basic hybrid stepping motor principle 


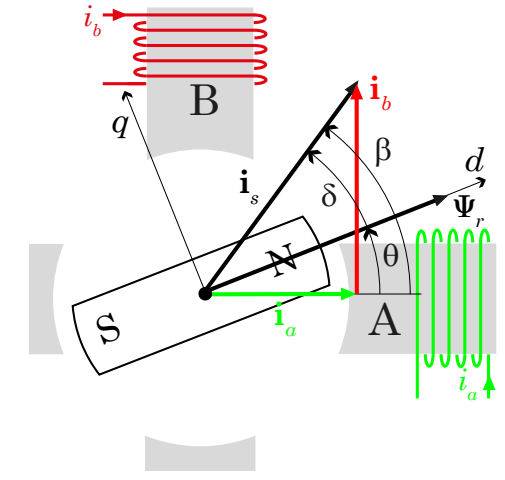

Figure 2: Current and flux vectors in the $d q$-reference frame fixed to the rotor flux $\Psi_{r}$

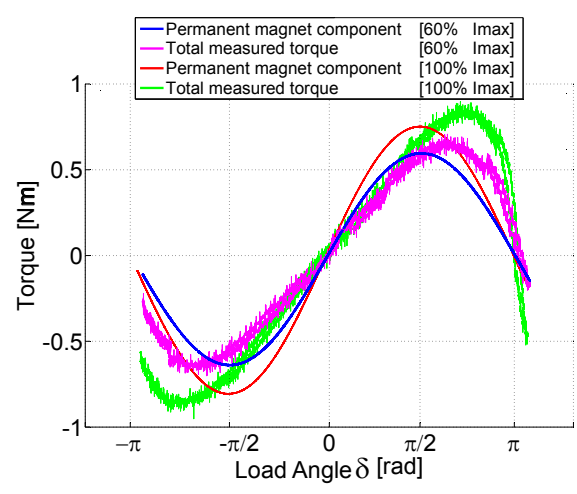

Figure 3: Measured torque load angle relation for $60 \%$ and $100 \%$ nominal current $I_{\max }$

\section{Stepping Motor Torque Generation}

The electromagnetic torque can be quantified by a vector based on the interaction between the stator flux linkage space vector $\Psi_{s}$ and the stator current space vector $\mathbf{i}_{s}[4]$.

$$
\mathbf{T}_{\text {motor }}=\Psi_{s} \times \mathbf{i}_{S}
$$

By neglecting saturation, the stator flux linkage space vector $\Psi_{S}$ can be written as the sum of the stator flux linkages, established by the two stator currents and the permanent-magnet rotor flux $\Psi_{r}$. In the $d q$-reference frame fixed to the rotor flux, illustrated in Fig. 2, the electromagnetic torque can be written as:

$$
\mathbf{T}_{\text {motor }}=\left(\Psi_{r}+\mathbf{i}_{d} \cdot L_{d}+\mathbf{i}_{q} \cdot L_{q}\right) \times \mathbf{i}_{s}
$$

The electromagnetic torque value can be written as a function of $i_{s}$ and the load angle $\delta$, defined as the angle between $\mathbf{i}_{s}$ and the rotor flux $\Psi_{r}$ :

$$
T_{\text {motor }}=\psi_{r} \cdot i_{s} \cdot \sin (\delta)+\frac{L_{d}-L_{q}}{2} \cdot i_{s}^{2} \cdot \sin (2 \delta)
$$

The first term in (3) describes the torque generated by the interaction between the permanent-magnet rotor flux $\Psi_{r}$ and the stator current $\mathbf{i}_{s}$. This term depends on the sine of the load angle $\delta$. Because of the multi toothed rotor and stator construction of a hybrid stepping-motor, the reluctance effect will increase the maximal electromagnetic torque. This reluctance effect is represented by the second term in (3) and varies sinusoidally with twice the load angle $\delta$. To quantify both effects, the motor torque is measured, using the test rig depicted in Fig. 16, at different positions while the rotor is blocked. The load angle $\delta$ is modified by changing the phase current set-points $i_{a}^{*}$ and $i_{b}^{*}$. For a current amplitude of $60 \%$ and $100 \%$ of the nominal current, measurement results are given in Fig. 3.

\section{Stepping Motor Drive Algorithms}

The construction of a stepping motor is ideally suited for open- loop positioning. When constant phase currents are applied, the rotor moves from one discrete steady-state position to another. By counting the step command pulses, open-loop positioning is achieved. A simulation is set-up to identify the impact of the stepping motor drive algorithm on the torque behavior. In the simulation model, the second term in equation (3) is neglected which is common in stepping motor motion control literature [5]. The mechanical load is modelled as an inertia $J$, a damping $b$ and a constant load torque $T_{\text {load }}$ as indicated in Fig. 8, the data used in the model is depicted in Fig. 16. This model is used to simulate the torque and load angle behaviour for different drive algorithms.

\section{Full-Step}

For the full-step algorithm, as illustrated in Fig. 5, only one phase at a time is excited. When a full-step command is given, the excitation of one phase is released while another phase is excited. The angular 
electric position $\beta$ of the current vector $\mathbf{i}_{s}$ changes with $\pi / 2$ at every full-step command, meaning the following pattern for $\beta$ :

$$
\beta=[0, \pi / 2, \pi, 3 \pi / 2,0+2 \pi, \pi / 2+2 \pi, \pi+2 \pi, 3 \pi / 2+2 \pi, \ldots]
$$

Incrementing $\beta$ gives rise to an abrupt change of the load angle $\delta$ and the motor torque $T_{\text {motor }}$ both characterized by a large ripple. This results in rather large steps of the mechanical angular position $\theta$.

\section{Half-Step}

When both phases are excited simultaneously (Fig. 7), a stable operating point 1 between 0 and 2 is obtained. The $\beta$ increment is halved compared to the full-step operation, resulting in the following pattern:

$$
\beta=[0, \pi / 4, \pi / 2,3 \pi / 4, \pi, 5 \pi / 4,3 \pi / 2,7 \pi / 4, \ldots]
$$

To obtain the same rotational speed, these so called half-step commands have to come twice as fast. As the excitation for the half-step drive algorithm illustrated in Fig. 7 alters between a maximum current in one phase and maximum current in two phases, the maximum torque is either $100 \%$ or $141 \%$ as illustrated by the length of the vector in the right part of Fig. 7. Because of this maximum torque variation, the drive algorithm proposed in Fig. 7 results in a more vibrating and noisy behaviour as indicated in Fig. 8.

However, more recent stepping motor drivers allow to adjust the current setpoint to obtain a constant maximum torque ( Fig. 9). This half-step algorithm results in a reduced torque ripple and less vibrating behavior as illustrated in Fig. 10.

\section{Micro-Step}

Finally, micro-stepping (Fig. 11) is based on additional current setpoints which are a fraction of the maximum current. This approach makes it possible to further reduce the step angle. As the step angle decreases, the movement becomes smoother as indicated in Fig. 12. Comparing Fig. 6 with Fig. 12 shows that a finer micro- stepping drive algorithm results in a much smoother operation. Fig. 13 is obtained using a 1/256th micro-stepping algorithm diminishing the vibrating behavior of a stepping motor drive system.

\section{Torque Ripples Due To The Stepping Motor Construction}

To identify the impact of the stepping motor drive algorithm a simplified version of equation (3) was used in the previous section. However, to study the effect of the stepping motor construction the complete equation (3) is considered. The second term in this equation describes the reluctance effect which is based on the difference between the direct axis inductance $L_{d}$ and the quadrature axis inductance $L_{q}$. Contant $L_{d}$ and $L_{q}$ values can be used if only the toothed rotor character of the rotor is considered. Hence, as illustrated in Fig. $1 \mathrm{~b}$ the stator surface is also toothed meaning both $L_{d}$ and $L_{q}$ depend on the angular rotor position [1]. Measurements in [6] show the inductance difference can be written as:

$$
L_{d}-L_{q}=-\Delta L+2 \cdot \bar{L} \cdot \sin \left(\theta_{\text {electrical }}\right)
$$

The block diagram of the simulation taking the toothed character of both rotor and stator into account is given in Fig. 14. To exclude the impact of the drive algorithm, instead of moving the the current vector $\tilde{\mathbf{i}}_{s}$ in discrete steps, $\mathbf{i}_{s}$ is given a constant rotational speed. The amplitude of the oscillation, caused by the toothed construction of rotor and stator, highly depends on the mechanical load (depicted in Fig. 16) and the speed setpoint. Fig. 15 shows simulation results at 190rpm. From this simulation it is clear that the toothed construction can cause significant torque ripples and corresponding load angle oscillations. The number of stator teeth determines the frequency of these oscillations $\omega_{\delta}$. Having $p$ stator teeth and a rotor speed of $\omega_{m} \mathrm{rad} / \mathrm{s}, \omega_{\delta}$ can be written as:

$$
\omega_{\delta}=p_{s} \cdot \omega_{m}
$$

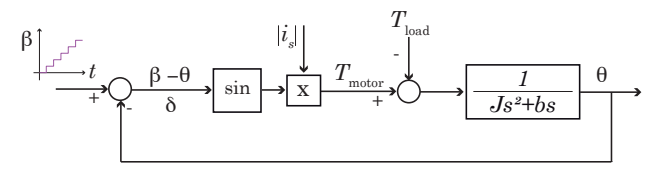

Figure 4: Diagram simulating the angular position, torque and load angle behavior 

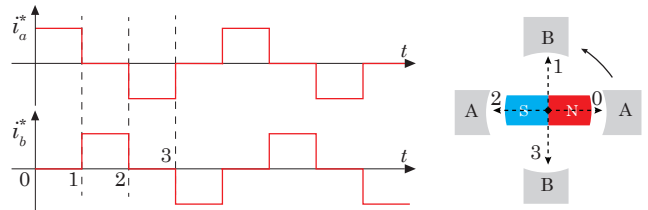

Figure 5: Full-step excitation scheme (left) and stable rotor positions (right)
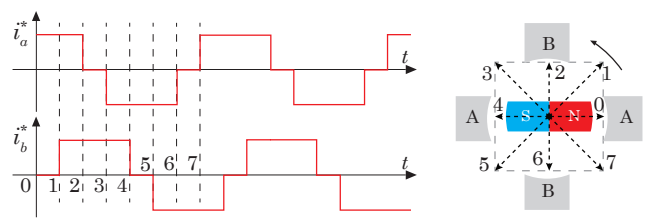

Figure 7: Half-step excitation scheme (left) and stable rotor positions (right)
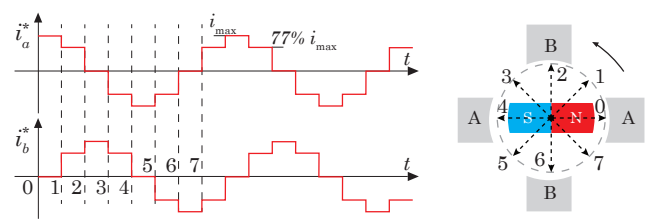

Figure 9: Compensated half-step excitation scheme (left) and stable rotor positions (right)


Figure 11: 1/4th micro-stepping excitation scheme (left) and stable rotor positions (right)

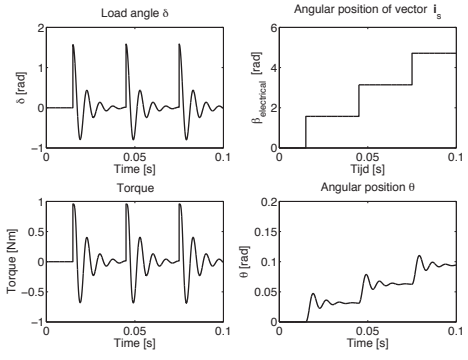

Figure 6: Simulated (according to Fig. 4) behavior of a full-step drive algorithm

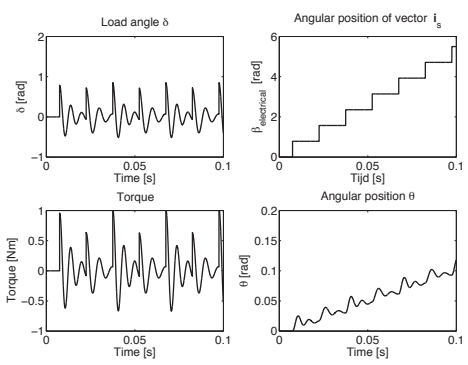

Figure 8: Simulated (according to Fig. 4) behavior of a half-step drive algorithm
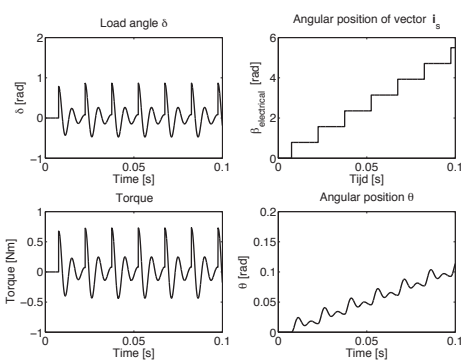

Figure 10: Simulated (according to Fig. 4) behavior of a compensated half-step drive algorithm
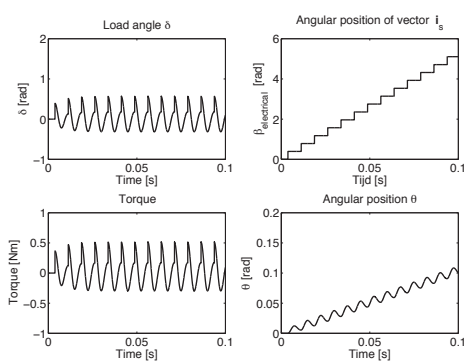

Figure 12: Simulated (according to Fig. 4) behavior of a 1/4th micro-stepping drive algorithm

\section{Measurements}

Load angle oscillations implies torque ripples, vibrations and noise. To characterize these vibrations the variance of the load angle $\operatorname{Var}(\boldsymbol{\delta})$ is used:

$$
\operatorname{Var}(\boldsymbol{\delta})=\frac{1}{n} \cdot \sum_{i=1}^{n}\left(\boldsymbol{\delta}-\boldsymbol{\delta}_{\text {mean }}\right)^{2}
$$



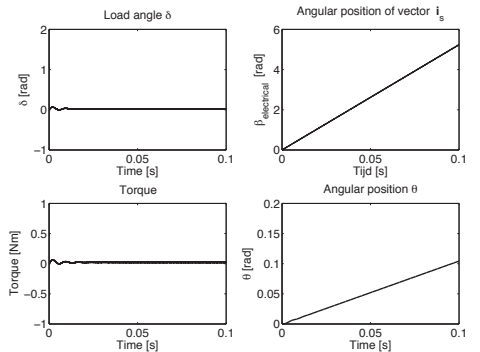

Figure 13: Simulated (according to Fig. 8) behavior of a very fine micro-stepping drive algorithm
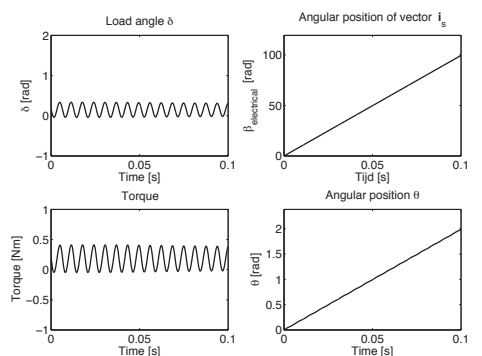

Figure 15: Simulations results at a constant speed of 190rpm according to Fig. 14 taking inductance variation into account

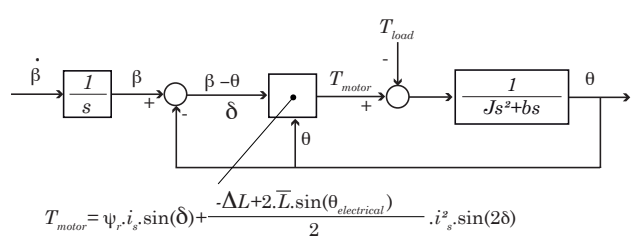

Figure 14: Block schematic representation of the angular position, torque and load angle behavior for a toothed rotor and stator construction

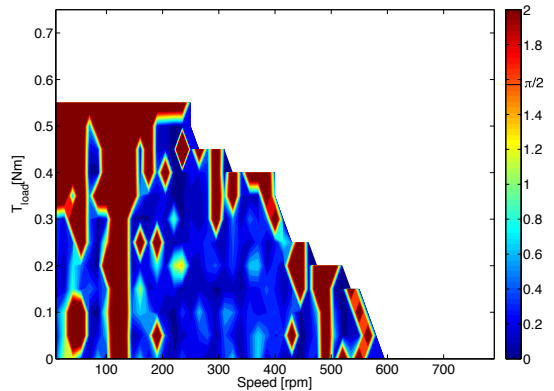

Figure 17: Measured load angle oscillations using a full-step drive algorithm

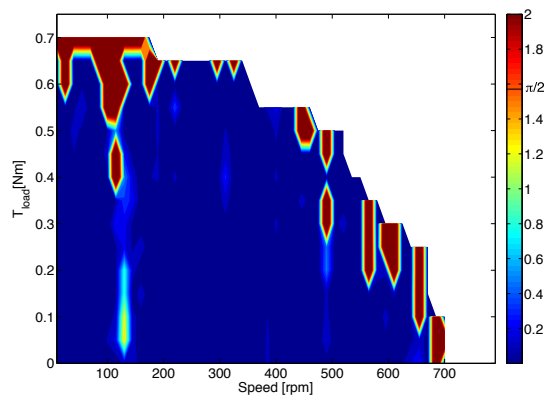

Figure 19: Measured load angle oscillations using a 1/4th micro-step drive algorithm

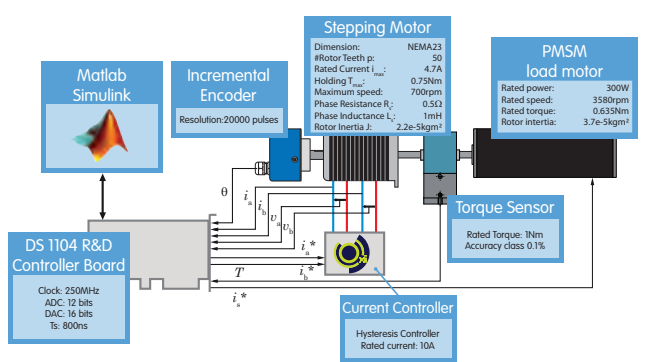

Figure 16: Test-rig

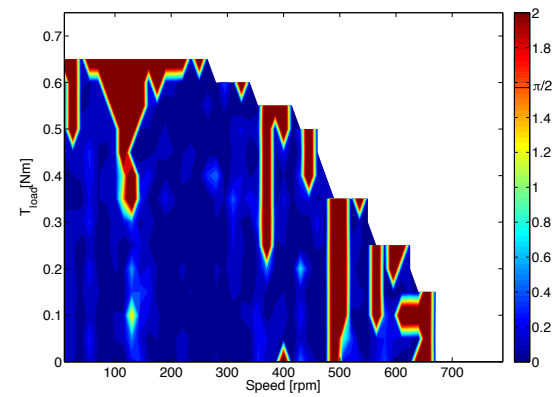

Figure 18: Measured load angle oscillations using a half-step drive algorithm

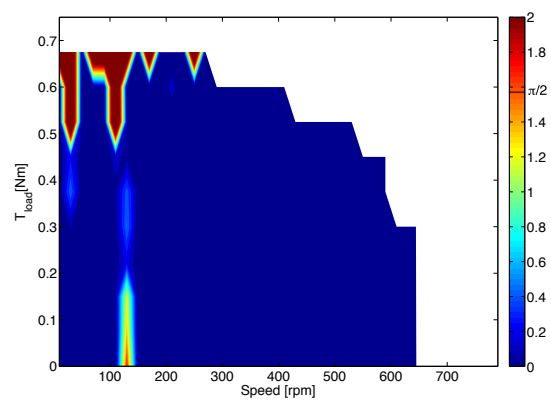

Figure 20: Measured load angle oscillations using a very fine micro-step drive algorithm

$\operatorname{Var}(\boldsymbol{\delta})$ is measured at different speeds and load torques for 4 different drive-algorithms. Speed setpoints are changed in steps of $2 \%$ of $n_{\max }$ while the load torque is incremented with $6 \%$ of $T_{\max }$. The results are depicted in Fig. 17 to Fig. 20 where different colors are used to indicate the level of vibrations. 
Fig. 3 indicates the maximum generated motor torque is available at a load angle of approximately $\pi / 2$ $\mathrm{rad}$. This means, heavy torsional vibrations leading to load angle values exceeding $\pi / 2 \mathrm{rad}$ result in steploss. The areas where this phenomenon occurs are indicted in Fig. 17 to Fig. 20 in deep red. This implies the stepping motor cannot be driven with the given algorithm at these speed setpoints and load torques. Fig. 17 shows that vibrations and the resulting step-loss seriously limits the operating range in full-step.

As indicated by the simulations in Figs. 6, 8, 10,12, 13 these vibrations are decimated when a full-step is divided in more micro-steps. However, even when the rotational speed $\dot{\beta}$ of the current excitation vector is constant speed as in Fig. 20 the toothed rotor and stator construction causes torsional vibrations. After a step command pulse the rotor oscillates, as seen in Fig. 13, with a frequency of 108Hz. This means, if the frequency of the step command pulses is $108 \mathrm{~Hz}$ or a multiple of it, resonance will occur. These resonances, visible at $130 \mathrm{rpm}$ in Fig. 17 to Fig. 20 can result in step-loss. Especially for full- and halfstep these resonance frequencies result in an uncontrollable stepping motor at those speed setpoints.

\section{Conclusion}

Typical problems for an open-loop stepping motor drive system include a large torque ripple, vibrations and a noisy operation. In this paper the two main causes of these torque ripples are discussed. First of all the drive algorithms using discrete displacements of the current excitation vector can result in a vibrating behavior. As a full-step is divided in more fine micro-steps the torque-ripples are heavily reduced as proven in this paper both by simulations and measurements. However, the toothed stator and rotor construction of a hybrid stepping motor also contributes to the torque ripples. This effect is explained in this paper based on the torque equation. Measurements and simulations for a 1/256 micro-stepping algorithm show these vibrations are significant at certain operating points.

\section{References}

[1] C. Kuert, M. Jufer, and Y. Perriard, "New method for dynamic modeling of hybrid stepping motors," in Conference Record of the Industry Applications Conference, 37th IAS Annual Meeting, vol. 1, 2002, pp. 6-12.

[2] M. Bodson, J. S. Sato, and S. R. Silver, "Spontaneous speed reversals in stepper motors," IEEE Transactions on Control Systems Technology, vol. 14, no. 2, pp. 369-373, 2006.

[3] K. Inaba, Y. Noda, T. Miyoshi, K. Terashima, M. Nishida, and N. Suganuma, "Driving control considering torsional vibration suppression in a stepping motor with a full-step drive," in Annual IEEE Industrial Electronics Conference. IECON, 2009, pp. 1116-1121.

[4] P. Vas, Sensorless vector and direct torque control. New York: Oxford University Press, 1998.

[5] S. M. Yang and E. D. A. M. D. O. t. Kuo, "Damping a hybrid stepping motor with estimated position and velocity," IEEE Transactions on Power Electronics, vol. 18, no. 3, pp. 880-807, 2003.

[6] S. Derammelaere, B. Vervisch, F. De Belie, J. Cottyn, G. Van den Abeele, P. Cox, K. Stockman, and L. Vandevelde, "A nonlinear and linear model of a hybrid stepping motor," in ELECTRIMACS, 2011. 